\title{
Chinese Herbal Medicine to Alleviate Vasomotor Symptoms in Breast Cancer Survivors: A Study Protocol for a Randomised, Double Blind, Placebo Controlled, Multi Centre, Cross Over Trial
}

\author{
Dianna Porter ${ }^{1 *}$, Xiaoshu Zhu ${ }^{1}$, Ping Ping Li ${ }^{2}$, Paul De Souza ${ }^{3}$, Paul Fahey ${ }^{4}$ and Alan Bensoussan ${ }^{1}$ \\ ${ }^{1}$ NICM Health Research Institute, Western Sydney University, Australia \\ ${ }^{2}$ Department of Beijing University Cancer Hospital, Beijing, China \\ ${ }^{3}$ Department of School of Medicine, Australia \\ ${ }^{4}$ Department of Statistician, School of Science \& Health, Western Sydney University, Australia
}

*Corresponding author: Dianna Porter, Department of NICM Health Research Institute, Western Sydney University, Australia.

To Cite This Article: Dianna Porter, Xiaoshu Zhu, Ping Ping Li, Paul De Souza, Paul Fahey, et al, Chinese Herbal Medicine to Alleviate Vasomotor Symptoms in Breast Cancer Survivors: A Study Protocol for a Randomised, Double Blind, Placebo Controlled, Multi Centre, Cross Over Trial. Am J Biomed Sci \& Res. 2020 - 7(1). AJBSR.MS.ID.001117. DOI: 10.34297/AJBSR.2020.07.001117.

Received: 此 December 18, 2019; Published: 海 January 28, 2020

\begin{abstract}
Introduction

Vasomotor Symptoms (VMS) have been documented as one of the most common and distressing side-effects after breast cancer treatment. Currently there is no standard conventional medical treatment. VMS, which include hot flushes and night sweats, can be the result of: a) treatmentschemotherapy, ovarian ablation, ovarian suppression and/or endocrine therapy with tamoxifen or aromatase inhibitors; b) menopause occurring naturally with a breast cancer diagnosis; and/or c) menopause symptoms occurring after cessation of hormone replacement therapy upon breast cancer diagnosis.
\end{abstract}

There is promising evidence of the use of a Chinese herbal medicine formula, Shu-Gan-Liang-Xue Decoction (SGLXD), developed by one author, PPL, for VMS in breast cancer survivors prescribed tamoxifen in China. The aim of this study is to evaluate whether SGLXD is an effective and safe treatment option for reducing VMS and improving quality of life in breast cancer survivors in Australia.

\section{Methods/Design}

We will conduct a multi-centre, randomised, double-blind, placebo controlled, cross-over trial. Participants are women, diagnosed with Stage I-III breast cancer, who have completed the acute stage of breast cancer treatment, who satisfy the inclusion/exclusion criteria and desire an intervention for VMS. Participants will be randomly allocated to treatments to create two groups for 12-weeks. After a 4-week wash-out period there will be a second 12-week period for which groups swap treatments. The primary outcome is reduction in frequency of VMS and secondary outcomes are reduction in mean severity of VMS (measured with subjective Hot Flush Daily Diary) and improvement in mean quality-of-life-score (Menopause Specific Quality of Life and Hot Flush Related Daily Interference Scale).

Safety will be measured with pathology tests including - liver and renal function tests, hormone assays, tamoxifen metabolites, Estrogen Receptor 1 Gene (ESR1), Circulating Tumour Cells (CTC) and adverse events log. At the end of the study focus groups will be conducted with study participants to discuss a range of topics including reasons for joining the study, experience of taking the intervention, effects of the treatment. Transcripts of the groups will be analysed using grounded theory techniques.

\section{Ethics and Dissemination}

This protocol has been approved by the South Western Sydney Local Health District Human Research Ethical Committee (Project number HE/17/013). All participants will receive Participant Information Sheets and verbal information about the trial and will give informed consent before enrolment. The results will be published in peer-reviewed journals and disseminated through conference presentations. 


\section{Trial Registration}

Australian New Zealand Clinical Trials Registry identifier: ACTRN12617001247369, Therapeutic Goods Administration, Cancer Institute of NSW Portfolio Trial C12017-852; Pre-results.

\section{Strengths and Limitations of this Study}

We are performing a randomised, double-blind, placebo controlled, cross-over trial to explore the safety and efficacy of a Chinese herbal medicine to alleviate vasomotor symptoms after breast cancer. The study builds on previous studies in Beijing with Chinese breast cancer survivors. Safety measures will include adverse effects log and pathology tests (including Liver and Renal function and hormone assays).The study is conducted in a primary care setting under the guidance of the referring oncologists and radiation oncologists and with the support of the clinical trials units at each hospital

\section{Introduction}

The number of women diagnosed with breast cancer is growing worldwide $[1,2]$. Due to improved detection methods and treatment the number of survivors is also increasing [3]. However, after treatment for the disease, up to $78 \%$ of survivors report bothersome vasomotor symptoms [4-7]. Vasomotor symptoms (VMS) include hot flushes and night sweats. A hot flush is a rushing sensation of heat spreading over the body and often accompanied by perspiration and a change in body temperature and heart rate [8]. VMS can also be accompanied by other physical sensations including palpitations, sweating, skin reddening, chills, tingling sensations in the extremities, dizziness and can disturb sleep leading to fatigue and mood changes [8]. Emotional symptoms can include panic, anxiety, frustration, irritation, depression and annoyance [8]. VMS can be a source of embarrassment and impact on quality of life for the individual can be distressing $[9,10]$. The higher frequency in breast cancer survivors can be due to age at diagnosis, abrupt discontinuation of Hormone Replacement Therapy (HRT) after diagnosis, chemotherapy, ovarian ablation or estrogen deficiency symptoms by therapy (tamoxifen or aromatase inhibitors) [11].

Studies have shown VMS to be the most frequently reported $[6,10]$ and residual symptom in the post treatment phase [10]. VMS can have a long-lasting negative effect on quality of life, and for some is an indicator for early discontinuation and compliance to life-saving endocrine therapies, putting them at increased risk of recurrence [4,6,12-17]. Many management options for VMS in healthy subjects have limited safety and effectiveness or adverse effects in this group of women. Hormone Replacement Therapy (HRT), the main management option used in healthy menopausal women, is contraindicated due to increased risk of recurrence of the disease $[18,19]$. Other treatments commonly prescribed include Selective Serotonin Re-Uptake Inhibitors (SSRIs), Serotonin Norepiephrine Re-Uptake Inhibitors (SNRIs), tiboline, gabapentin, clonidine and stellate ganglion block [20-23].

These interventions can induce negative side-effects or have only moderate effect [23-25]. This leaves clinicians with uncertainty about how to treat VMS safely and effectively, after breast cancer [5,26-28]. Given the absence of a safe and effective treatment intervention, many cancer patients turn to some form of complementary medicine [29-34].The aim of the study is to a) evaluate the effectiveness of the Chinese herbal medicine (SGLXD) on reduction of VMS and improvement in quality of life in Australian women treated for breast cancer, b) to monitor the safety of $S G L X D$ in this group of women, and c) to explore the feasibility of implementing and evaluating a Chinese herbal medicine intervention within an Australian primary care system (with regard to recruitment patterns, compliance, withdrawal rate).

\section{Rationale for Use of Chinese Herbal Medicine}

Chinese herbal medicine is a popular and prevalent therapy worldwide, in individuals diagnosed with cancer to alleviate treatment related side-effects and to improve quality of life [3538]. However, clinical evidence on the efficacy and safety of Chinese herbal medicine for VMS in breast cancer survivors outside of China is limited $[3,30]$.

\section{Previous Studies}

Shu-Gan-Liang-Xue Decoction (SGLXD) was modified from the classical formula Dan-Zhi-Xiao-Yao-San widely used in treating menopausal disorders and described in the Traditional Chinese Medicine (TCM) canon "Neike Zhaiyao" (Summary of Internal Medicine) in the Ming Dynasty (A.D.1368-1644) [39]. In TCM rationale SGLXD belongs to the syndrome of "liver soothing and blood cooling" actions [39]. SGLXD granules are composed of the following herbs: paeonia suffruticosa, Schisandra chinensis, Paeonia lactiflora, bupleurum chinense with vinegar, curcumae wenjuyin, arnebia euchroma, cynanchum atratum. The herbal formula is designated to have the following properties according to modern pharmacology: anti-inflammatory, anti-pyretic, anti-tumour, immune modulating, CNS suppressant, neuroprotective [40-68]. The standardised SGLXD formula has been used by Chinese breast cancer patients for over a decade [69]. Individualised Chinese herbal medicine formula is 'gold standard' in traditional Chinese medicine practice [70]. Everyone is prescribed a different formula, which may alter over time, based on their presenting signs and symptoms [70]. 
Standardised formulae are when every participant is prescribed the same herbal formula without considering their presenting signs and symptoms $[70,71]$. The standardized formula makes the results of the trial more generalisable [70]. Data from laboratory studies and clinical trials conducted in China have shown the effectiveness of SGLXD to ameliorate VMS associated with treatment of breast cancer in women and improvement in sleep quality [72-74]. SGLXD also decreased serum estrogen and alleviated the effects of tamoxifen on endometrial hypertrophy in vivo [75]. A study found that SGLXD inhibited breast tumour growth by $26.3 \%$ in tumour-bearing mice [76]. Another study, by Zhang and Li, 2009, found the herbal formula and each of the component herbs did not manifest any estrogenic activity and the formula exhibited an antiproliferative effect on MCF-7cell viability at high concentrations [39].

Appropriate safety of SGLXD has been shown in herb-drug safety/interaction studies [39, 59, 73, 76-89]. This preliminary research has been limited to China, with Chinese breast cancer survivors and in a Chinese hospital setting. Building on these pre-clinical and clinical results we propose a trial to evaluate the clinical effectiveness and safety of SGLXD in Australian women in an Australian primary care setting. As Chinese herbal medicine is culturally ingrained in China $[38,90]$, this study may ascertain feasibility and tolerability in Australian breast cancer survivors and provide important outcomes on the use of CHM in primary care. This novel intervention for VMS may therefore translate to increased compliance to endocrine therapy and improved quality of life for a growing cohort of Australian survivors.

\section{Methods}

\section{Design}

The study design is a multi-centre, randomised, double-blind, placebo-controlled, cross-over clinical trial comparing SGLXD and placebo granules in participants with VMS after breast cancer treatment. The study schema is represented in (Figure 1). The protocol design is based on the Standard Protocol Items: Recommendations for Interventional Trials (SPIRIT) Checklist (File 1). The study complies with the principles of the Declaration of Helsinki and Good Clinical Practice guidelines [91, 92].

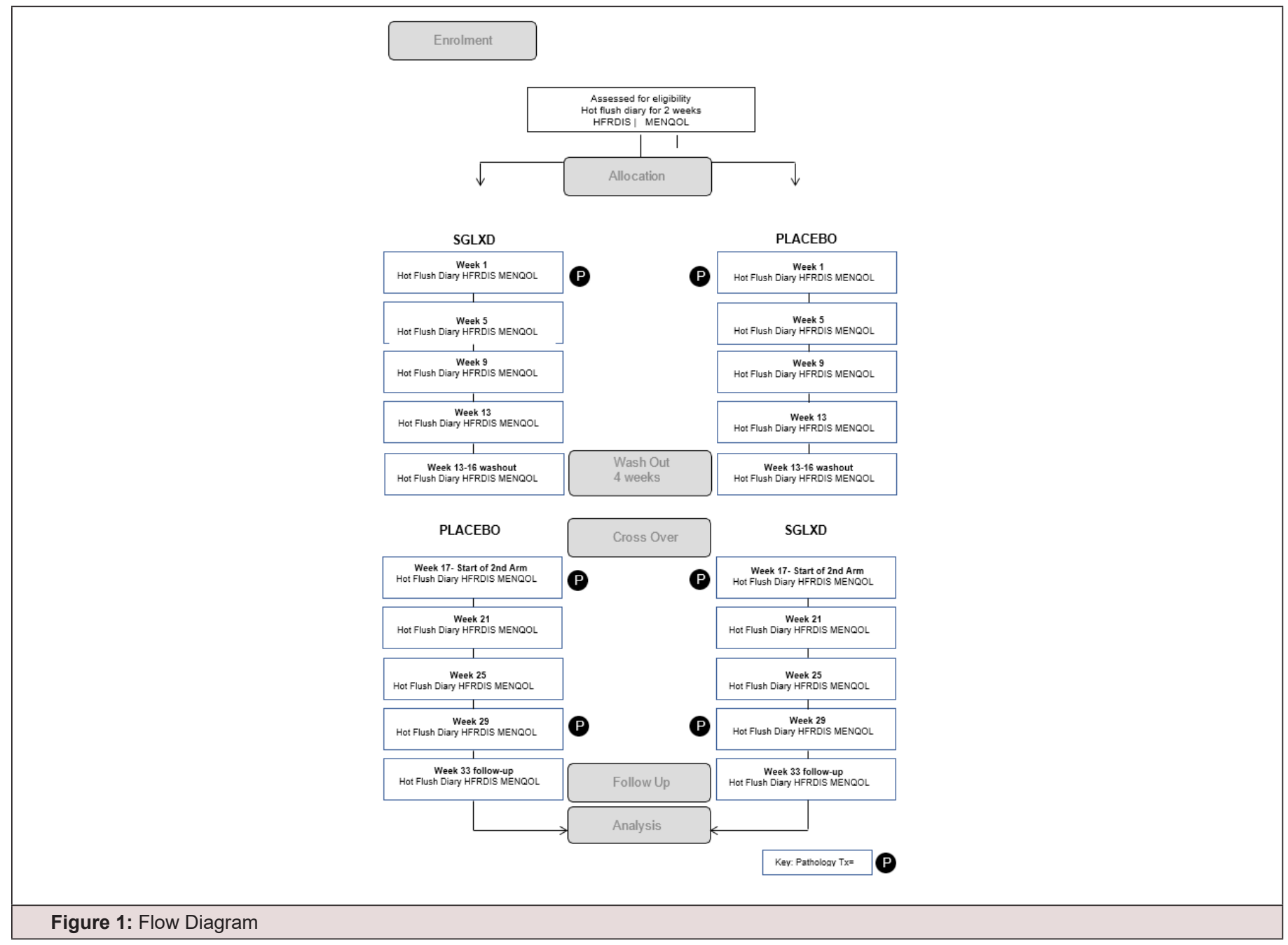




\section{Ethics and Trial Registration}

This protocol has been approved by the South Western Sydney Local Health District Human Research Ethical Committee (Project number HE/17/013). All participants will receive Participant Information Sheets and verbal information about the trial and will give informed consent before enrolment. Any amendments to the study protocol will be submitted to the HREC of SWSLHD for approval. The trial has been registered with the Australia and New Zealand Clinical Trials Register (ANZCTR) ACTRN12617001247369, the Clinical Trial Notification (CTN) Scheme, the Therapeutic Goods Administration (TGA) and the Cancer Institute of New South Wales Portfolio Trial C12017-852.

\section{Setting}

Eighty-four participants will be recruited from three hospitals in South Western Sydney, Australia. All sites are under the governance of the South Western Sydney Local Health District which provided publicly funded health services to over 820,000 people. The hospitals service an area regarded as one of the most culturally diverse in Australia with more than 38\% speaking a language other than English at home [93].

\section{Eligibility Criteria and Enrolment}

Inclusion/exclusion criteria have been designed to rule out systemic co-morbidities and pharmaceuticals/over the counter remedies that may cause or interfere with vasomotor symptoms. Women will be eligible if 1) they have been diagnosed with stage I-III breast cancer, 2) completed surgery, chemotherapy and/or radiation, 3) if prescribed an endocrine therapy i.e. tamoxifen or aromatase inhibitor, stable dosage for $>2$ months prior to the study, 4) unlikely to be changing/discontinuing endocrine therapy during study, 5) with minimum of at least 3 hot flushes/24 hours during the eligibility period prior to randomisation (Table 1 ).

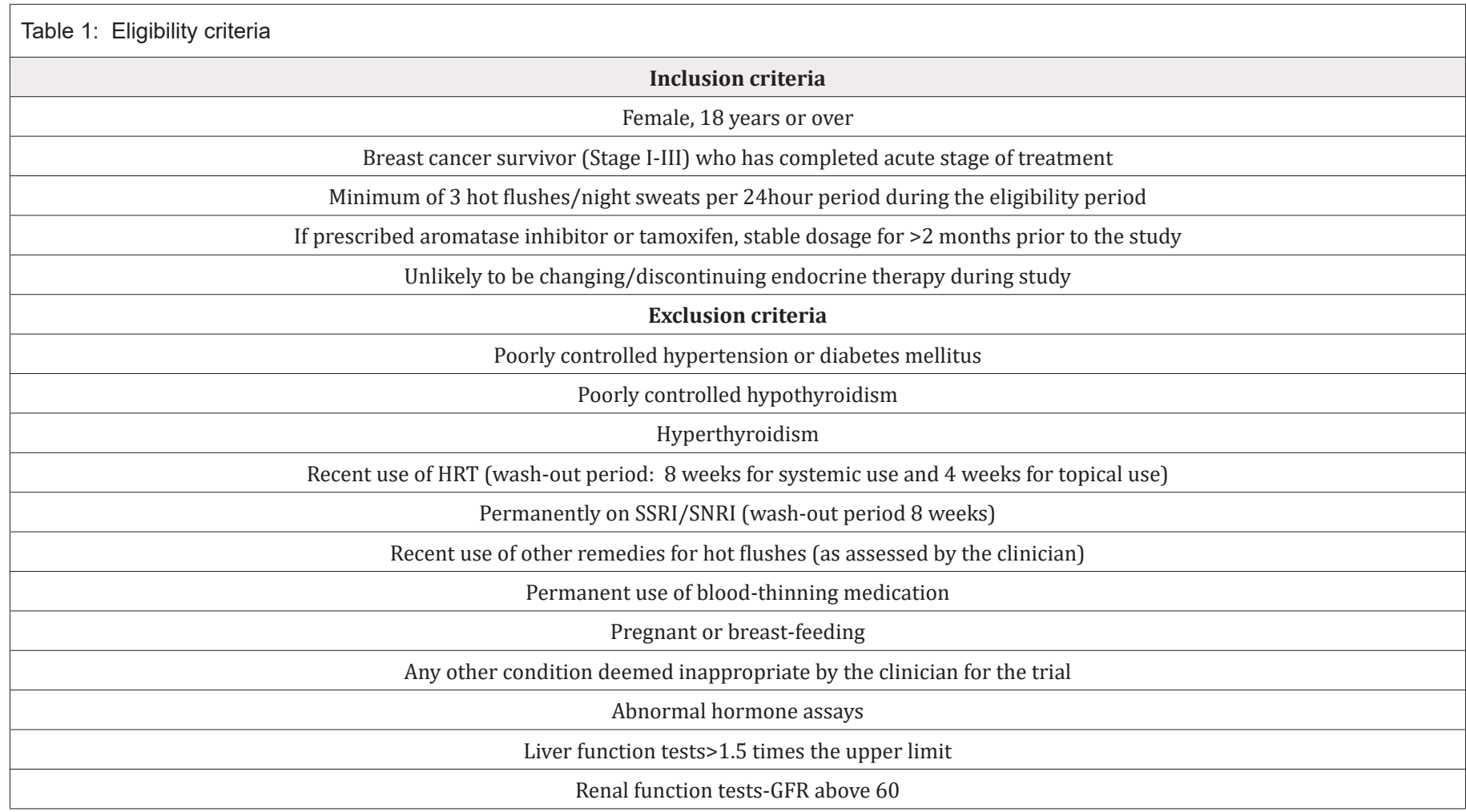

Women will be excluded if 1) they have poorly controlled hypertension, hypothyroidism or diabetes mellitus, 2) hyperthyroidism, 3) liver function test $>1.5$ times upper limit of normal, 4) renal function test - Glomerular Filtration Rate (GFR) below 60,5) use of Hormone Replacement Therapy (HRT) within the previous 8 weeks for systemic and 4 weeks topical/local use, 6) use of SSRI/SNRI (wash-out period 8 weeks), 7) use of other remedies for hot flushes during study, deemed inappropriate by the clinician for the trial 8) currently pregnant or breast-feeding, 9) permanent use of blood-thinning medication, 10) any other chronic condition deemed inappropriate by the clinician for the trial (Table 1).

Potential participants will be identified via referring oncologists at the participating sites, based on the preliminary inclusion and exclusion criteria, and provides an information sheet outlining the study, their commitments and possible risks involved. They are then directed to the principal researcher who will explain the study and answer any questions. If they agree to join the study and after signed informed consent forms are obtained, they will be further 
screened to satisfy all inclusion/exclusion criteria, including pathology tests. The study consists of a 2 -week eligibility/baseline period, a 12-week treatment period, a 4-week wash-out period and cross-over to the other arm for a 12-week treatment period, followed by a 4-week follow-up period. The duration of the run-in, treatment and follow-up was decided based on a previous study [94]. An outline of procedures is illustrated in (Figure 1).

\section{Sub Study}

As an added safety measure, we will be conducting additional pathology testing on a small cohort of women prescribed endocrine disrupting drugs. Additional consent is required from women who volunteer to participate. Sub-study (a) is for women prescribed tamoxifen and sub-study (b) is for women prescribed aromatase inhibitors. The sub-study will conduct tests at the same three timepoints as the standard pathology tests.

A. To ascertain if the herbal medicine has an impact on plasma concentration of tamoxifen metabolites.

B. Acquired Estrogen Receptor 1 gene (ESR1) mutations are found predominately in women diagnosed with estrogen receptor positive breast cancer and exposed to aromatase inhibitors [95]. Mutations of the ESR1 gene, the gene that encodes the estrogen receptor, have been recognised as a potential mechanism of endocrine therapy resistance and as an experimental predictive biomarker to guide therapeutic decisions [96]. Testing may provide valuable information on whether the herbal medicine impacts ESR1 mutations. Circulating Tumour Cells (CTCs) characteristics may serve as future indicators of therapeutic interventions [97].

\section{Randomisation and Blinding}

If all eligibility criteria are confirmed participants will be randomised. Randomisation will be conducted external to the primary research team by a research officer at the NICM Health Research Institute. A computer generated, permuted block randomisation sequence will randomly associate a unique identifier to either active or placebo treatment (1:1 ratio) and for labelling the codes on the pre-prepared participant medication containers. Permuted block randomisation (14 per block) will ensure that the treatment groups are balanced at the end of every block. Each participant will be assigned a Personal Identification Number (PIN) at baseline and once randomised will also be provided a randomisation number.

Both sets of codes will be documented and stored securely at the NICM and this information will also be secured on the University Research Data Storage system. This can be accessed by the research officer at NICM, when required, for un-blinding. Throughout the study, the participants, doctors, researchers and the data analyst will be blind to the current treatment of participants until after the study is completed.

\section{Trial Interventions}

All trial participants will receive Chinese herbal medicine granules (SGLXD) or placebo in powder form (indistinguishable to each group).The herbal medicine and placebo are manufactured as a concentrated herbal extract by Pura Pharm, Hong Kongin accordance with Australian TGA (Therapeutic Goods Administration) Good Manufacturing Practice (GMP) requirements for herbal medicinal products and according to the Product Specification File [98]. The medication will be mixed, cooked, filtered and pressure-spraydried forming granules. For the control group, placebo granules are prepared from therapeutically inert ingredients -calcium hydrogen phosphate $(78.2 \%)$, soy fibre $(19.6 \%)$, natural identical liquorice flavour (1\%), bitter flavour $(0.3 \%)$, colours $(0.9 \%)$.

Dosage and administration instructions for both groups will be identical. Each intervention, in a water-soluble form, will be administered in a standard 10gram dose, orally twice per day for each 12-week intervention period. Each dose is packaged individually in a sealed foil sachet. Participants dissolve the granules in hot water to take as a tea. Interventional Medical Product (IMP) is packaged as 4-weeks supply (i.e. 56 sachets) to be dispensed at each study visit. The package contains the randomisation number and the batch number and no information as to the contents. The placebo arm is packaged identical to the control arm. Participants will be asked to return all IMP sachets at the next scheduled visit, including those that are empty. Unused IMP will be counted and logged to assess compliance.

\section{Outcome Measures}

\section{Primary outcome}

The primary outcome is the changes or differences in VMS frequency, as recorded subjectively in the Hot Flush Daily Diary. Frequency will be averaged over a week (week -1, 4, 8, 12, 16, 20, $24,28,32$ ) and assessed for change across time and between active and placebo treatment.

\section{Secondary Outcome}

The secondary outcomes of the study will assess the changes or differences across time between the intervention group and the placebo group.

A. Mean severity score of vasomotor symptoms as measured by the Hot Flush Daily Diary (HFDD) during active treatment minus the change in mean severity score of VMS during the placebo treatment

B. To assess change across time in Menopause Specific Quality of Life Questionnaire Scores (MENQoL)

C. To assess change across time in hot flush interference as measured by the Hot Flush Related Daily Interference Scale (HFRDIS) 
D. Treatment acceptability measured with Treatment Satisfaction Score at the end of each treatment arm and focus group qualitative data collected at the end of the study.

\section{Safety Endpoints}

A. Number and kind of adverse events will be monitored at each review visit by a clinician according to Acute Toxicity CT CAE version 4.0 assessment form (99)

B. Changes in serum hormone levels (FSH, LH, E2, progesterone), liver and renal function will be monitored during the study at 3 time-points. Tamoxifen metabolites $(<10)$, ESR1and Circulating Tumour Cells (CTC) $(<20)$ will be monitored in a small cohort of women at the same 3 time-points.

\section{Qualitative Data}

Focus groups will be conducted at the end of the study for those who initially volunteered and consented to participate in a group discussion These groups of up to 15 participants will run for approximately 30 minutes and will be digitally recorded to obtain qualitative data on participants' subjective experiences of joining the study, including their response to the taste, burden, compliance and effect of taking the intervention. This may provide valuable information on the feasibility of integrating CHM with conventional care.

\section{Assessment Schedules}

After a 2-week eligibility period, the study period will run for $2 \times 12$-week periods with a 4-week wash-out period in between (Table 2). Baseline demographic and clinical data will be collected including age, age at diagnosis, type and stage of cancer, ER positive/negative, node positive/negative, treatment (surgery, chemotherapy, radiation, endocrine therapy), medical history, menopausal status, height, weight, country of birth, current medications, serum hormone levels, liver and renal function test results, TCM diagnosis. Potential participants will fill in the HFDD for 2-weeks prior to randomisation to establish eligibility criteria and baseline data. After randomisation the HFDD will be filled out for Weeks 1-4, Week 8, 12, Weeks 17-20, Week 24, 28, 32. Other clinical data including HFRDIS, MENQoL, Adverse Effects log and a Medication Log (to note any changes to current medications) will be collected during the eligibility period and end of Weeks 5, 9, 13 (end of first arm,) week 17 (end of wash-out period), and end of Weeks 21, 25, 29 (end of second arm), with follow-up at the end of Week 33 (Table 2).

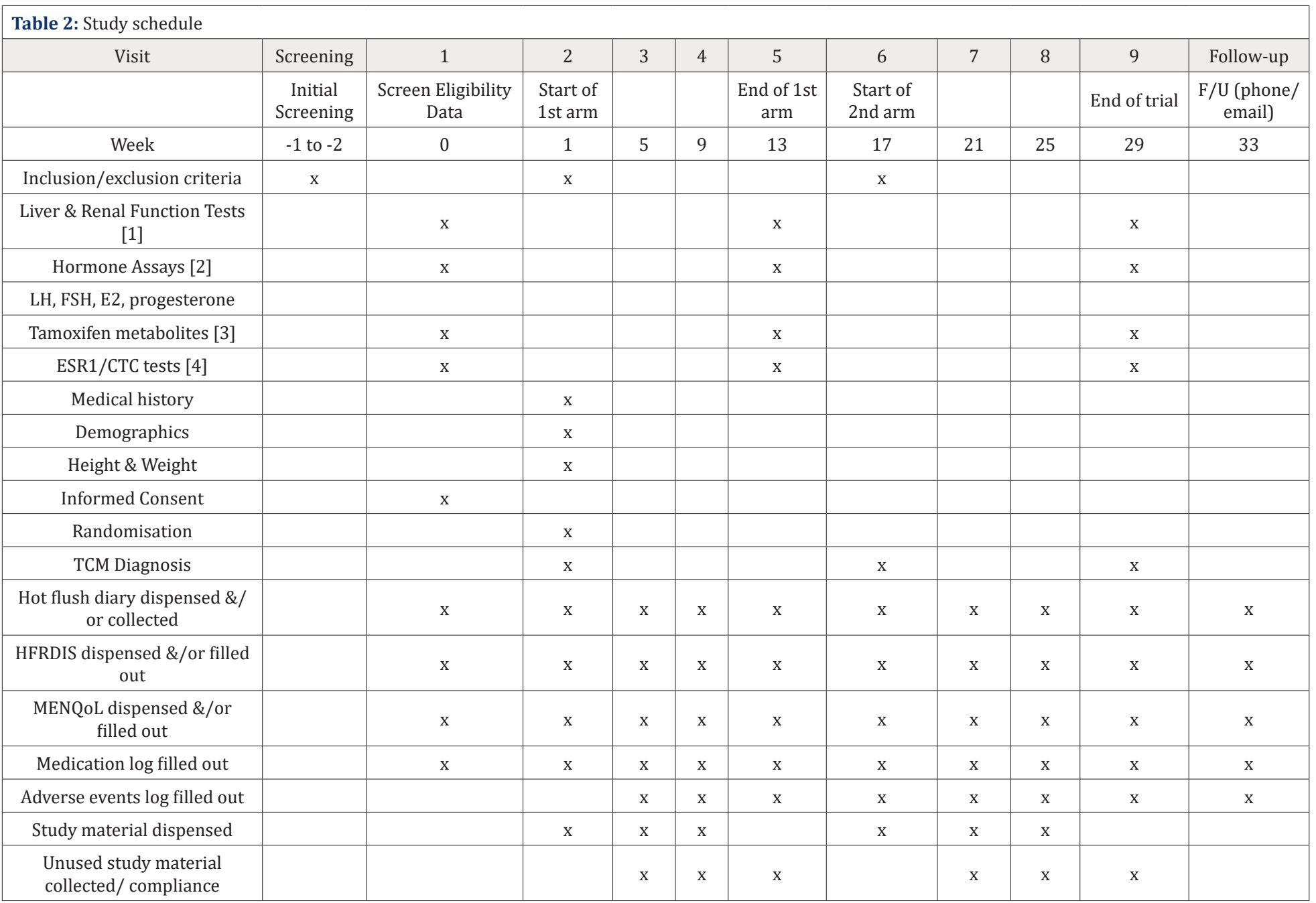


This should permit measurement of any short and longerterm effects on quality of life. At the end of each arm a Treatment Satisfaction form will be filled in by each participant, detailing their satisfaction on a rating scale of $0-5$, with 1 being not satisfied and 5 being very satisfied. All data will be de-identified and securely stored at the hospitals and at the NICM. The Hot Flush Daily Diary, a subjective symptom diary, previously validated in numerous breast cancer hot flush studies, was chosen to measure the primary outcome (frequency). Additionally, the Hot Flush Daily Diary records the severity of hot flushes/night sweats and can be used to calculate score by multiplying the number of hot flushes with their severity [99-102].This diary rates VMS severity numerically according to 4 categories: mild, moderate, severe and very severe from the participants' perception. For each 24-hour period the participant subjectively records the frequency of every VMS experienced in each of the categories.

The Hot Flush Related Daily Interference Scale (HFRDIS) is a validated tool that has been used in previous breast cancer trials $[103,104]$. The HFRDIS rates the impact of VMS on a 10-point scale on 10 domains: including work, leisure, social activities, sleep and quality of life as a whole [104].The Menopause Specific Quality of Life (MENQoL) questionnaire is used to compare impact of treatment arms on quality of life [105-107]. Its psychometric properties have been validated in a sample of breast cancer survivors experiencing menopausal symptoms [105]. It consists of a 29-item, condition-specific quality of life instrument covering domains of physical, emotional and social aspects which defines if a therapeutic intervention affects an individual's quality of life specific to menopause symptoms. Participants are asked about symptoms and rate the problem on a seven-point Likert Scale. Scores range from 0-6, with lower scores indicating better quality of life. The MENQoL and HFRDIS will be used as secondary endpoints.

The MENQoL and the HFRDIS are questionnaires designed for self-assessment of impact of hot flushes on daily life and overall quality of life and can be used for monitoring treatment effects [104, 107] In this study they will be used to make baseline assessments as well as effectiveness measures. Both can be completed in approximately 5 minutes.

\section{Safety Monitoring and Emergency Un-blinding}

An independent safety monitor has been appointed. The safety monitor has relevant expertise (Bachelor of Medicine, $\mathrm{PhD}$ in Pharmacology) and will assess any Serious Adverse Events (SAEs) or Suspected Unexpected Serious Adverse Reaction (SUSARs). Liver and renal function tests and hormone assays will be conducted at baseline, to establish eligibility, and at the end of each intervention for all participants ( 3 collection times). Hormone assays will include FSH, LH, estrogen, progesterone. Tamoxifen metabolites will be tested, in a small cohort of women $(<10)$ prescribed tamoxifen, at baseline and end of each treatment arm. Circulating Tumour
Cells (CTC) and Estrogen Receptor 1 (ESR1) testing will also be conducted in a small cohort of women $(<20)$ prescribed tamoxifen or aromatase inhibitors as a further safety measure.

If any adverse events are reported appropriate treatment will be provided and documented in a case report form, including severity, duration and probable causality. All SAEs and SUSARs must be recorded in detail in the Clinical Report Forms (CRF) and reported to the Chief Investigator and appointed Safety Monitor within 24 hours. Emergency un-blinding will be escalated by the appointed Medical Monitor for a Serious Adverse Event (SAE) or a Suspected Unexpected Serious Adverse Reaction (SUSAR). The TGA will be notified within 7-15 days. Follow-up for SAEs/SUSARs will be provided until resolution of the event.

\section{Sample Size}

The sample size calculation was performed using G*Power using data from previous trials $[94,108]$. Allowing for a $25 \%$ drop in frequency of hot flushes due to placebo effect we define minimum clinically important improvement to be twice the size of the placebo effect (50\% decrease in frequency) [94, 109]. We expect the mean frequency to be 6.0 per day with a standard deviation of 4 at baseline [94]. We estimate the required sample size is 58 to produce $80 \%$ power to detect the difference between $25 \%$ decrease (placebo) [110] and 50\% decrease (treatment), using a two-sided matched pairs t-test with $5 \%$ significance level. Assuming an attrition rate of $30 \%$ (withdrawal and non-compliance), based on earlier research, a target sample of 84 participants is required [111].

\section{Data Collection and Management}

Case Report Forms (CRF) from this study will be in paper and electronic format for all participants. Data will be collected at various time points (Table 2). Baseline data collected will cover demographics and clinical history including age, type and stage of breast cancer, age at diagnosis, treatment undertaken, menopausal status, current medications, height and weight, smoking/alcohol status, 2-week Hot Flush Daily Diary (HFDD), Hot Flush Related Daily Interference Scale (HFRDIS), Menopause Specific quality of Life Questionnaire (MENQoL) and pathology test results. Follow-up data will include HFDD, HFRDIS, MENQoL, Concomitant Medication Log, Adverse Effects Log, pathology test results, Treatment Satisfaction Form. All paper questionnaires will be self-administered and collected by the principal investigator at each visit. All participants will be given a unique Participant Identification Number (PIN) to maintain confidentiality. All Clinical Report Forms (CRFs), pathology tests and reports will be identified with the participant's PIN. All trial data will be entered on an electronic data capture system (Redcap-Research Electronic Data Capture) an online secure web-based application initiated by Vanderbilt University [112]. All data collected will be retained for 15 years in the Western Sydney University Research Data Repository where it will be securely archived. 


\section{Data Checking}

At completion of data collection and all variables and all logical pairs of variables will be subject to descriptive analyses using graphs, frequency counts and summary statistics. This will allow a) identification of unusual or unexpected results for data checking and b) familiarisation with the distributions and associations within the data set. Outcome variables which have severely nonsymmetric distributions will be either transformed or categorised. At the completion of data checking and correction, the data set will be locked and remain blinded until completion of the primary and secondary endpoint analysis.

\section{Statistical Analysis}

The demographic and medical characteristics of participants will be summarised using percentages or means and standard deviations. To address withdrawals, loss to follow-up or noncompliance with the protocol, the study analyses will be conducted on both an Intention to Treat (ITT) and Per Protocol (PP) basis. The ITT population is defined as all participants who are recruited into the study and receive the treatment sachets. Withdrawing and non-compliant participants shall be encouraged to continue with data collection even if stopping treatment. Where data items are missing, we shall use the last value carried forward method to replace missing data within each 12 -week treatment block in the ITT analysis. Participants who have significant deviations from the protocol will be removed from the PP analysis after the completion of the ITT analysis. Any deviation from the protocol, missing data and withdrawals will be fully reported.

Between group differences from baseline to the end of each treatment will be undertaken applying independent samples $t$ tests. Linear mixed models (with participant as the random effect) will be used to analyse changes over time. Results will be reported with a 95\% confidence interval. Data will be analysed using Statistical Package for the Social Sciences (SPSS software). All tests will be two-sided, and $\mathrm{p}$-value $<0.05$ will be considered statistically significant. Analyses are conducted blinded to group allocation. The main outcomes of reducing the frequency and severity of VMS will be analysed. The primary analysis is intention-to-treat principle with baseline frequency and treatment order as predictors. We will also describe changes in quality of life, adherence to IMP, adverse effects, retention of participants. Numbers and reasons for withdrawal will be recorded as these are key feasibility outcomes that may inform a larger trial.

\section{Discussion}

\section{Strengths of The Study}

\section{Regulatory approval}

The trial has been approved by the Human Research Ethics Committee of South Western Sydney Local Health District and will be conducted in a hospital setting with all participants referred and initially screened by a participating oncologist. The oncology clinical trial units at each site will assist with co-ordination of the study. This study may help establish a precedent for subsequent CHM trials within primary care.

\section{Crossover study}

The trial is designed as a cross-over trial which involves two consecutively administered treatments for each participant. A 2-week pre-treatment period is used to establish eligibility and the baseline frequency/severity of VMS. After the first treatment period a wash-out period of 28 days was given to ensure that any residual effect from the first treatment did not linger and effect the second treatment. Advantages of cross-over trials are they are statistically efficient (less participants required to achieve the same level of statistical power). It also reduces between participant variability as each participant serves as their own control (treatment A versus treatment B on the same participant) and is suitable for chronic conditions where we are not looking for a cure but alleviating symptoms and improving quality of life and for separating treatment effects from period effects $[113,114]$.

\section{Safety}

Safety of the concentrated herbal granules is an import issue for this study. Purapharm's manufacturing process adheres to strict regulatory and quality control of Good Manufacturing Process (GMP) [98]. The placebo is also manufactured by PuraPharm to be similar in taste, colour, smell and appearance and is packaged identically to the intervention. Adverse events will be logged at each visit, by the oncologist or a hospital staff member not otherwise involved in the study, to reduce bias. The appointed medical monitor has authority to stop the trial if there are serious adverse events related to the intervention. Additionally, safety is also monitored with hormone assays, liver and renal function tests on all participants. (Table 2).

\section{Collaborating organizations}

This project will bring together practitioners of Chinese Medicine, in Australia and China, in collaboration with oncologists and clinical trials units from South Western Sydney Local Health District. In summary the trial design and management are of a high standard, due to the involvement of the medical and radiation oncologists and the radiation/oncology clinical trial unit personnel. This involvement developed a consensus-based trial protocol with ethical approval from the SWSLHD HREC. We have also had the input of TCM oncologists and CHM researchers in China and all these factors point to a rigorous and professional study using herbal medicine that meets the standard for GMP. Additional scientific scrutiny (tamoxifen metabolite testing/ESR1/CTCs) may help develop guidelines for future CHM investigations.

A further strength of this study is that it extends knowledge further than all previous studies in the field as it includes the following: 
A. The current study is a randomised, double blind, placebocontrolled, cross-over study whereas the previous study was observational.

B. While the previous study only recruited participants experiencing vasomotor symptoms as a side-effect of Tamoxifen the current study will recruit participants experiencing vasomotor symptoms for any reason after breast cancer treatment.

In addition to measuring hot flushes the current study will measure quality of life and hot flush related interference in daily activities and conduct focus groups to obtain qualitative data.

\section{Potential limitations of the study}

One potential limitation of this study is participants selfreporting their VMS. Subjective measures can be inaccurate especially with reporting of night sweats. However, researchers at the Mayo Clinic conducted several VMS trials and argue that the diaries were reliable and consistent [99]. The second limitation is that each participant receives the same formula. Usually in Chinese medicine everyone receives a diagnosis according to the symptoms and signs at that time and formulae are adjusted accordingly. Although they all have the same western medical diagnosis, i.e. VMS after breast cancer treatment, we shall conduct a TCM diagnosis to see whether a TCM sub-group or groups respond more effectively than other diagnoses.

Thirdly, due to our inclusion criteria results will only be generalisable to women with enough English language proficiency without co-morbidities. If results from this study are promising, follow-up trials of these other groups may be warranted. Management of VMS in breast cancer survivors can be challenging for clinicians and so an alternative approach may be an important a therapeutic option. The findings may contribute to achieving a novel intervention for alleviating VMS after breast cancer and, thereby, increased quality of life for a growing number of survivors and may develop future Chinese herbal medicine research methodology.

\section{Trial Status}

Recruitment commenced in January 2018 and is ongoing.

\section{Abbreviations}

$\begin{array}{lll}\text { AE } & : & \text { Adverse events } \\ \text { ANZCTR } & : & \begin{array}{l}\text { Australian New Zealand Clinical } \\ \text { Trials Registry }\end{array} \\ \text { CHM } & : & \text { Chinese herbal medicine } \\ \text { CNS } & : & \text { Central nervous system } \\ \text { CRF } & : & \text { Clinical report forms } \\ \text { CTC } & : & \text { Circulating Tumour Cells } \\ \text { CTN } & : & \text { Clinical Trial Notification }\end{array}$

\begin{tabular}{|c|c|c|}
\hline E2 & : & Estrogen \\
\hline ER & : & Estrogen receptor \\
\hline ESR1 & : & Estrogen Receptor 1 Gene \\
\hline FSH & : & Follicle Stimulating Hormone \\
\hline GMP & : & Good Manufacturing Process \\
\hline HFDD & : & Hot Flush Daily Diary \\
\hline HFRDIS & : & $\begin{array}{l}\text { Hot Flush Related Daily Interference } \\
\text { Scale }\end{array}$ \\
\hline HREC & : & Human Research Ethics Committee \\
\hline HRT & : & Hormone Replacement Therapy \\
\hline IMP & : & Investigational Medicinal Product \\
\hline ITT & : & Intention to treat \\
\hline $\mathrm{LH}$ & : & Luteinizing Hormone \\
\hline MENQoL & : & $\begin{array}{l}\text { Menopause Specific Quality of Life } \\
\text { Questionnaire }\end{array}$ \\
\hline NICM & : & NICM Health Research Institute \\
\hline PIN & : & Participant identification number \\
\hline PP & : & Per protocol \\
\hline Redcap & : & Research Electronic Data Capture \\
\hline SAE & : & Serious adverse event \\
\hline SERM & : & Selective estrogen receptor modulator \\
\hline SGLXD & : & Shu-Gan-Liang-Xue Decoction \\
\hline SNRI & : & $\begin{array}{l}\text { Serotonin norepinephrine re-uptake } \\
\text { inhibitor }\end{array}$ \\
\hline SSRI & : & Selective serotonin re-uptake inhibitor \\
\hline SUSAR & : & $\begin{array}{c}\text { Suspected Unexpected Serious } \\
\text { Adverse Reaction }\end{array}$ \\
\hline SWSLHD & : & $\begin{array}{l}\text { South Western Sydney Local Health } \\
\text { District }\end{array}$ \\
\hline TGA & : & Therapeutic Goods Administration \\
\hline TCM & : & Traditional Chinese Medicine \\
\hline VMS & : & Vasomotor symptoms \\
\hline
\end{tabular}

\section{Competing Interests}

The authors declare that they have no competing interests. Professor Li developed the CHM formula.

\section{Authors Contributions}

DP, XZ, AB and PDS designed the RCT with advice from the participating oncologists. PF was consulted to calculate sample 
size for the study and for advice on outcomes and data analysis. DP and the clinical trials units will manage all aspects of the trial including, recruitment, screening, administration, processing and analysis, data analysis, reporting and communication of findings to participants. XZ, AB and PDS will supervise the clinical trial. DP wrote the initial draft of the manuscript and XZ, PL and PF were co-authors. All authors have contributed to, read and approved the final manuscript.

\section{Acknowledgement}

The authors acknowledge Dr Kellie Bilinski for preparation and concealment of the randomisation schedule, Professor Stephen Myers for his role as Safety Monitor, PuraPharm, the manufacturer of $S G L X D$, for providing the trial medications. The company will not be involved in any other aspect of trial design or implementation. Funding for this research has been received in the format of an Australian post-graduate award scholarship, of which DP is a recipient, research training funds provided by Western Sydney University as a standard resource to all higher degree research candidates, a top-up scholarship award from Centre of Oncology Education and Research Translation (CONCERT) of which DP is a recipient and additional trial funding from the NICM Health Research Institute. The authors would like to acknowledge the Ingham Institute and Westmead Millennium Research Centre for their assistance with the tamoxifen metabolite tests and CTC/ESR1 tests. This trial is part of a PhD for DP and results will be published.

\section{References}

1. Current Statistics in Australian Breast Cancer.

2. Ferlay J, Soerjomataram I, Dikshit R, Eser S, Mathers C, et al. (2015) Cancer incidence and mortality worldwide: Sources, methods and major patterns in GLOBOCAN 2012. International Journal of Cancer 136(5): 359-386.

3. Antoine C, Liebens F, Carly B, Pastijn A, Rozenberg S (2007) Safety of alternative treatments for menopausal symptoms after breast cancer: a qualitative systematic review. Climacteric 10(1): 23-26.

4. Adelson K, C Loprinzi, DL Hershman (2005) Treatment of hot flushes in breast and prostate cancer. Expert Opin Pharmacother 6(7): 1095-1106.

5. Cella D, Fallowfield LJ (2008) Recognition and management of treatment-related side effects for breast cancer patients receiving adjuvant endocrine therapy. Breast cancer research and treatment 107(2): 167-180.

6. Hickey M, Saunders C, Stuckey B (2005) Management of menopausal symptoms in patients with breast cancer: an evidence-based approach. The lancet oncology 6(9): 687-695.

7. Parkin D M, Bray F, Ferlay J, Pisani P (2005) Global cancer statistics, 2002. CA a Cancer journal for clinicians 55(2): 74-108.

8. Morrow PKH, Mattair DN, Hortobagyi GN (2011) Hot Flashes: A Review of Pathophysiology and Treatment Modalities. Oncologist 16(11): 16581664 .

9. Barba M, Pizzuti L, Sergi D, Sacca MM, Vincenzoni C, et al. (2014) Hot flushes in women with breast cancer: state of the art and future perspectives. Expert Review of Anticancer Therapy 14(2): 185-198.

10. Gupta P, Sturdee DW, Palin SL, Majumder K, Fear R, et al. (2006) Menopausal symptoms in women treated for breast cancer: the prevalence and severity of symptoms and their perceived effects on quality of life. Climacteric 9(1): 49-58.

11. Bordeleau L, Pritchard K, Goodwin P, Loprinzi C (2007) Therapeutic options for the management of hot flashes in breast cancer survivors: an evidence-based review. Clinical therapeutics 29(2): 230-241.

12. Barron TI, Connolly R, Bennett K, Feely J, Kennedy MJ (2007) Early discontinuation of tamoxifen. Cancer 109(5): 832-839.

13. Coates AS, Keshaviah A, Thürlimann B, Mouridsen H, Mauriac L, et al. (2007) Five years of letrozole compared with tamoxifen as initial adjuvant therapy for postmenopausal women with endocrineresponsive early breast cancer: update of study BIG 1-98. Journal of Clinical Oncology 25(5): 486-492.

14. Demissie S, Silliman R, Lash T (2001) Adjuvant tamoxifen: Predictors of use, side effects, and discontinuation in older women. J Clin Oncol 19(2): 322-328.

15. Fellowes D, Fallowfield LJ, Saunders CM, Houghton J (2001) Tolerability of hormone therapies for breast cancer: how informative are documented symptom profiles in medical notes for well-tolerated' treatments? Breast cancer research and treatment 66(1): 73-81.

16. Makubate B, Donnan PT, Dewar JA, Thompson AM, Mc Cowan C (2013) Cohort study of adherence to adjuvant endocrine therapy, breast cancer recurrence and mortality. Br J Cancer 108(7): 1515-1524.

17. Sestak I, Kealy R, Edwards R, Forbes J, Cuzick J (2006) Influence of hormone replacement therapy on tamoxifen-induced vasomotor symptoms. Journal of clinical oncology 24(24): 3991-3996.

18. Sismond P, Kimmig R, Kubista E, Biglia N, Egberts J, et al. (2011) Effects of tibolone on climacteric symptoms and quality of life in breast cancer patients-Data from LIBERATE trial. Maturitas 70(4): 365-372.

19. Santen RJ, Allred DC, Ardoin SP, Archer DF, Boyd N, et al. (2010) Postmenopausal Hormone Therapy: An Endocrine Society Scientific Statement. The Journal of Clinical Endocrinology \& Metabolism 95(7): $1-66$.

20. Guttuso T (2013) Stellate ganglion block for treating hot flashes: A viable treatment option or sham procedure? Maturitas 76(3): 221-224.

21. Fenlon D (2011) Clonidine SSRIs, SNRIs and gabapentin reduce hot flushes in women with a history of breast cancer -

22. relaxation therapy may have a mild effect in the short term. Evidence Based Nursing 14(2): 62.

23. (2007) Venlafaxine vs clonidine for hot flushes in breast cancer patients. Inpharma Weekly 1594(1): 17.

24. Rada G, Capurro D, Pantoja T, Corbalán J, Moreno G, et al. (2010) Nonhormonal interventions for hot flushes in women with a history of breast cancer. Cochrane Database of Systematic Reviews 8(9): 4923.

25. Othman AH, Zaky AH (2014) Management of Hot Flushes in Breast Cancer Survivors: Comparison Between Stellate Ganglion Block and Pregabalin. Pain Medicine 15(3): 410-417.

26. Lipov EG, Joshi JR, Xie H, Slavin KV (2008) Updated findings on the effects of stellate-ganglion block on hot flushes and night awakenings. Lancet Oncology 9(9): 819-820.

27. Hsieh K, Chen LC, Cheung KL, Chang CS, Yang YH (2014) Interruption and Non-Adherence to Long-Term Adjuvant Hormone Therapy Is Associated with Adverse Survival Outcome of Breast Cancer Women An Asian Population-Based Study. PLoS One 9(2): 87027.

28. Partridge A (2006) Non-adherence to endocrine therapy for breast cancer. Ann Oncol 17(2): 183-184.

29. Partridge A, Wang PS, Winer EP, Avorn J (2003) Nonadherence to adjuvant tamoxifen therapy in women with primary breast cancer. J Clin Oncol 21(4): 602-606. 
30. Chen Z, Gu K, Zheng Y, Zheng W, Lu W, et al. (2008) The Use of Complementary and Alternative Medicine Among Chinese Women with Breast Cancer. J Altern Complement Med 14(8): 1049-1055.

31. Cui Y, Shu XO, Gao Y, Wen W, Ruan ZX, et al. (2004) Use of complementary and alternative medicine by Chinese women with breast cancer. Breast Cancer Research Treatment 85(3): 263-270.

32. Henneghan AM, Harrison T (2015) Complementary and Alternative Medicine Therapies as Symptom Management Strategies for the Late Effects of Breast Cancer Treatment. Journal of Holistic Nursing 33(1): 84-97.

33. Lu W, Clower ED, Gilman AD, Rosenthal DS (2008) The Value of Acupuncture in Cancer Care. Hematology Oncology Clinics of North America 22(4): 631-648.

34. Ramsay NA, Kenny MW, Davies G, Patel JP (2005) Complimentary and alternative medicine use among patients starting warfarin. British journal of haematology 130(5): 777-780.

35. Sluijs C (2007) Complementary and alternative medicine use and the menopausal transition the effect of a Chinese herbal formula on vasomotor symptoms and bone turnover, M University of Western Sydney. Centre for Complementary, University of Western Sydney.

36. Wanchai A, Armer JM, Stewart BR (2010) Complementary and alternative medicine use among women with breast cancer: a systematic review. Clinical Journal of Oncology Nursing 14(4): 45-55

37. Buhling KJ, Daniels BV, Studnitz FS, Eulenburg C, Mueck AO (2014) The use of complementary and alternative medicine by women transitioning through menopause in Germany: Results of a survey of women aged 4560 years. Complementary Therapies in Medicine 22(1): 94-98.

38. Kronenberg F, Berman AF (2002) Complementary and alternative medicine for menopausal symptoms: a review of randomized, controlled trials. Annals of internal medicine 137(10): 805-813.

39. Liu J, Wang S, Zhang Y, Fan HT, Lin HS (2005) Traditional Chinese medicine and cancer: History, present situation, and development. Thorac Cancer 6(5): 561-569.

40. Zhang Y, Li PP (2009) Evaluation of estrogenic potential of Shu-GanLiang-Xue Decoction by dual-luciferase reporter based bioluminescent measurements in vitro. Journal of Ethnopharmacology 126(2): 345-349.

41. Chen JK (2004) Chinese medical herbology and pharmacology. Gamble A, CramptonL (ed.). City of Industry Calif: Art of Medicine Press.

42. Bensky D (2015) Chinese herbal medicine : Materia medica. $3^{\text {rd }}$ ed. Materia medica. Bensky D, Clavey S, Stöger E, Seattle WA (ed.). Seattle, WA: Eastland Press, USA.

43. Wu M, Gu Z (2009) Screening of Bioactive Compounds from Moutan Cortex and Their Anti-Inflammatory Activities in Rat Synoviocytes. Evid based Complement Altern Med 6(1): 57-63.

44. Wong VK, T Li, B YK Law, E DL Ma, N C Yip, et al. (2013) Saikosaponin-d, a novel SERCA inhibitor, induces autophagic cell death in apoptosisdefective cells. Cell death disease 4(7): 720.

45. Wang R, Yin R, Zhou W, Xu D, Li S (2012) Shikonin and its derivatives: a patent review. Expert Opinion on Therapeutic Patents 22(9): 977-997.

46. Wan CK, Tse AK, Yu ZL, Zhu GY, Wang H, et al. (2010) Inhibition of cytochrome P450 3A4 activity by schisandrol A and gomisin A isolated from Fructus Schisandrae chinensis. Phytomedicine 17(8-9): 702.

47. Yuan PS, Zhou SF, Gao SH, Yu ZL, Zhang SF, et al. (2013) New Perspectives on How to Discover Drugs from Herbal Medicines: CAM's Outstanding Contribution to Modern Therapeutics. Evidence-Based Complementary and Alternative Medicine pp.25.

48. Prasad CP, Rath G, Mathur S, Bhatnagar D, Ralhan R (2009) Potent growth suppressive activity of curcumin in human breast cancer cells Modulation of Wnt/ $\boldsymbol{\beta}$-catenin signaling. Chemico biological interactions 181(2): 263-271
49. Pandya KJ, Morrow GR, Roscoe JA, Zhao H, Hickok JT, et al. (2005) Gabapentin for hot flashes in 420 women with breast cancer: a randomised double-blind placebo-controlled trial. The Lancet 366(9488): 818-824.

50. Nagaraju GP, Aliya S, Zafar SF, Basha R, Diaz R, et al. (2012) The impact of curcumin on breast cancer. Integrative Biology 4(9): 996-1007.

51. Law BYK, Mo JF, Wong VKW (2014) Autophagic effects of Chaihu (dried roots of Bupleurum Chinense DC or Bupleurum scorzoneraefolium WILD). Chinese medicine 9(1): 21 .

52. Kurien B, Scofield RH (2009) Curry spice curcumin and prostate cancer. Mol Nutr Food Res 53(7): 939-940

53. Korwek Z, Zmijewska AB, Mosieniak G, Alster O, Villanueva MM, et al. (2013) DNA damage-independent apoptosis induced by curcumin in normal resting human $\mathrm{T}$ cells and leukaemic Jurkat cells. Mutagenesis 28(4): 411-416

54. Kim J, Noh EM, Kwon KB, Kim JS, You YO, et al. (2012) Curcumin suppresses the TPA-induced invasion through inhibition of PKC alphadependent MMP-expression in MCF-7 human breast cancer cells. Phytomedicine 19(12): 1085-1092.

55. Jin G, Dai Y, Feng J, Qin X, Xue X, et al. (2010) 2-D RP/RPLC method to separate components in Fructus schisandrae chinensis. Journal of Separation Science 33(4-5): 564-569.

56. Ide H (2012) Prostate cancer and curcumin: current application and future prospect. Int J Urol 19: 59-59.

57. Hempen CH (2009) A materia medica for Chinese medicine : plants, minerals, and animal products. $1^{\text {st }}$ ed in English ed. TMD Fischer Edinburgh: Edinburgh : Churchill Livingstone.

58. He DY, Dai SM (2011) Anti-Inflammatory and Immunomodulatory Effects of Paeonia Lactiflora Pall, a Traditional Chinese Herbal Medicine. Frontiers in Pharmacology 2:10.

59. He C, Peng B, Dan Y, Peng Y, Xiao P (2014) Chemical taxonomy of tree peony species from China based on root cortex metabolic fingerprinting. Phytochemistry 107: 69-79.

60. Chen J, Chang NW, Chung JG, Chen KC (2003) Saikosaponin-A induces apoptotic mechanism in human breast MDA-MB-231 and MCF-7 cancer cells. Am J Chin Med 31(3): 363-377.

61. Yang J, Kim JS, Sa YJ, Kim MO, Jeong HJ, et al. (2011) Antioxidant antibacterial and [alpha]-glucosidase inhibitory activities of different extracts of Cortex Moutan. African Journal of Biotechnology 10(46): 9438-9444.

62. Yang H, Zhou P, Huang H, Chen D, Ma N, et al. (2009) Shikonin exerts antitumor activity via proteasome inhibition and cell death induction in vitro and in vivo. Int J Cance 124(10): 2450-2459.

63. Rath KS, McCann GA, Cohn DE, Rivera BK, Kuppusamy P, et al. (2013) Safe and targeted anticancer therapy for ovarian cancer using a novel class of curcumin analogs. Journal of Ovarian Research 6(1): 35.

64. Qiu FM, Zhong XM, Mao QQ, Huang Z (2013) Antidepressant-like effects of paeoniflorin on the behavioural, biochemical, and neurochemical patterns of rats exposed to chronic unpredictable stress. Neuroscience Letters 541: 209-213.

65. Furuya R, Hu H, Zhang Z, Shigemori H (2012) Suffruyabiosides A and B, Two New Monoterpene Diglycosides from Moutan Cortex. Molecules 17(5): 4915-4923.

66. Dhillon N, Aggarwal BB, Newman RA, Wolff RA, Kunnumakkara AB, et al. (2008) Phase II trial of curcumin in patients with advanced pancreatic cancer. Clinical Cancer Research 14(14): 4491-4499.

67. Chendil D, Ranga RS, Meigooni D, kumar SS, Ahmed MM (2004) Curcumin confers radiosensitizing effect in prostate cancer cell line PC3. Oncogene 23(8): 1599-1607. 
68. Chen X, Yang L, Zhang N, Turpin JA, Buckheit RW, et al. (2003) Shikonin, a component of Chinese herbal medicine, inhibits chemokine receptor function and suppresses human immunodeficiency virus type 1 . Antimicrob Agents Chemother 47(9): 2810-2816.

69. Buch Z, et al. (2012) Interventional role of Haridra (Curcuma longa Linn) in cancer. Clinical Cancer Investigation Journal 1(2): 45.

70. Zhang Y, PP Li (2010) Shu-Gan-Liang-Xue Decoction, a Chinese herbal formula, down-regulates the expression of steroid sulfatase genes in human breast carcinoma MCF-7 cells. Journal of ethnopharmacology 127(3): 620-624

71. Flower A, Witt C, Liu JP, Ulrich-Merzenich G, Yu H, et al. (2012) Guidelines for randomised controlled trials investigating Chinese herbal medicine. Journal of Ethnopharmacology 140(3): 550-554.

72. Flower A, Harman K, Lewith G, Moore M, Bishop FL, et al. (2016) Standardised Chinese herbal treatment delivered by GPs compared with individualised treatment administered by practitioners of Chinese herbal medicine for women with recurrent urinary tract infections (RUTI): study protocol for a randomised controlled trial. Trials 17(1): 358.

73. Sun $\mathrm{H}$, et al. (2009) Effect of shu gan lian xue compound for relieving hot flushes in breast cancer patients. Chinese Journal of Integrated Traditional and Western Oncology 29(1): 3.

74. Li P (2004) Effect of traditional Chinese medicine in improving hot flashes symptom of breast cancer. Chin J Integrat Med 10: 166-167.

75. Xue D, H Sun, Li PP (2011) Long-term Chinese herbs decoction administration for management of hot flashes associated with endocrine therapy in breast cancer patients. Chinese Journal of Cancer Research 23(1): 74-78.

76. Li PP, Wang W, Xie YQ (2003 ) In vivo effect of Shu-Gan-Liang-Xue decoction on estrogen. Zhonghua Zhong Liu Za Zhi 25(5): 445-447.

77. Wu CX,PP Li (2008) Anti-tumour effect of Shu-Gan-Liang-Xue Decoction combined with tamoxifen on estrogen-dependent breast cancer. Chinese Journal of Experimental Traditional Medical Formulae 14: 31-34.

78. Chen J, Xie J, Jiang Z, Wang B, Wang Y, et al. (2011) Shikonin and its analogs inhibit cancer cell glycolysis by targeting tumor pyruvate kinase-M2. Oncogene 30(42): 4297-4306.

79. Fu X, Li PP (2011) Shu-Gan-Liang-Xue decoction simultaneously downregulates expressions of aromatase and steroid sulfatase in estrogen receptor positive breast cancer cells. Chin J Cancer Res 23(3): 208-213.

80. Hung JY, Yang CJ, Tsai YM, Huang HW, Huang MS (2008) Antiproliferative activity of paeoniflorin is through cell cycle arrest and the Fas/Fas ligand-mediated apoptotic pathway in human non-small cell lung cancer A549 cells. Clin Exp Pharmacol Physiol 35(2): 141-147.

81. Li P, W Wang, Y Xie (2003) In vivo effect of Shu-Gan-Liang-Xue decoction on estrogen. Zhonghua zhong liu za zhi [Chinese journal of oncology] 25(5): 445-447.

82. Li X, Yang G, Li X, Zhang Y, Yang J et al. (2013) Traditional Chinese medicine in cancer care: a review of controlled clinical studies published in Chinese. PLoS One 8(4): 60338.

83. Papageorgiou VP, Assimopoulou AN, Couladouros EA, Hepworth D, Nicolaou KC (1999) The chemistry and biology of alkannin, shikonin, and related naphthazarin natural products. Angewandte Chemie International Edition 38(3): 270-301.

84. Singh F, Gao D, Lebwohl MG, Wei H (2003) Shikonin modulates cell proliferation by inhibiting epidermal growth factor receptor signaling in human epidermoid carcinoma cells. Cancer letters 200(2): 115-121.

85. Yao Y, Zhou Q (2010) A novel antiestrogen agent Shikonin inhibits estrogen-dependent gene transcription in human breast cancer cells. Breast Cancer Res Treat 121(1): 233-240.

86. Zhang Y, Li PP (2010) Shu-Gan-Liang-Xue Decoction, a Chinese herbal formula, down-regulates the expression of steroid sulfatase genes in human breast carcinoma MCF-7 cells. Journal of Ethnopharmacology 127(3): 620-624.

87. Zhang Y, Li PP (2011) Assessment of shikonin for potential estrogenic activity by dual-luciferase reporter based bioluminescent measurements in vitro. Pharmazie 66(2): 141-143.

88. Zhang Y, Qian R, Li PP (2009) Shikonin, an ingredient of Lithospermum erythrorhizon, down-regulates the expression of steroid sulfatase genes in breast cancer cells. Cancer Letters 284(1): 47-54.

89. Zhou F, Han S, Zhou N, Zheng W, Li P (2015) Effects of modified ShuGan-Liang-Xue decoction combined with anastrozole on osteoblastic proliferation and differentiation of MC3T3-E1 cells. Mol Med Rep 11(3): 1639-1646.

90. Zhou N, Han SY, Zhou F, Li PP (2014) Anti-tumor effect of Shu-gan-LiangXue decoction in breast cancer is related to the inhibition of aromatase and steroid sulfatase expression. J Ethnopharmacology 154(3): 687695.

91. Unschuld PU (2010) Medicine in China : a history of ideas. 25 anniversary. Berkeley (ed.). University of California Press pp.464.

92. World Medical Association (2013) World Medical Association Declaration of Helsinki: ethical principles for medical research involving human subjects. JAMA 310(20): 2191-2194.

93. (2018) The Australian Clinical Trial Handbook Do Health, Editor, Therapeutic Goods Administration: Australia South. Western Sydney Communities.

94. Kimmick GG, Lovato J, McQuellon R, Robinson E, Muss HB (2006) Randomized, Double-Blind, Placebo-Controlled, Crossover Study of Sertraline (Zoloft) for the Treatment of Hot Flashes in Women with Early Stage Breast Cancer Taking Tamoxifen. Breast J 12(2): 114-122.

95. Turner NC, et al. (2014) Tracking tumor-specific mutations in circulating-free DNA to predict early relapse after treatment of primary breast cancer. J Clin Oncol 32(15).

96. Fard AT, Srihari S, Ragan MA (2015) Breast cancer classification: linking molecular mechanisms to disease prognosis. Briefings in Bioinformatics 16(3): 461-474

97. Graves H, Czerniecki BJ (2011) Circulating tumor cells in breast cancer patients: an evolving role in patient prognosis and disease progression. Pathology Res Int 621090.

98. (2009) Guide to Good Manufacturing Practice for Medicinal Products Annexes. Pharmaceutical Inspection Co-operation Scheme: Geneva.

99. Sloan JA, Loprinzi CL, Novotny PJ, Barton DL, Lavasseur BI, et al. (2001) Methodologic lessons learned from hot flash studies. J Clin Oncology 19(23): 4280-4290.

100. Loprinzi CL, Barton DL, Rhodes D (2001) Management of hot flashes in breast-cancer survivors. Lancet Oncology 2(4): 199-204.

101. Buijs C, Mom CH, Willemse PH, Boezen HM, Maurer JM, et al. (2009) Venlafaxine versus clonidine for the treatment of hot flashes in breast cancer patients: a double-blind, randomized cross-over study. Breast Cancer Res Treat 115(3): 573-580.

102. Barton DL, Loprinzi C, Gostout B (2002) Current management of menopausal symptoms in cancer patients. Oncology 16(1): 67-72.

103. Rand KL, Otte JL, Flockhart D, Hayes D, Storniolo AM, et al. (2011) Modeling hot flushes and quality of life in breast cancer survivors. Climacteric 13(6): 171-180.

104. Carpenter JS (2001) The Hot Flash Related Daily Interference Scale: a tool for assessing the impact of hot flashes on quality of life following breast cancer. J pain symptom manage 22(6): 979-989.

105. Radtke JV, Terhorst L, Cohen SM (2011) The Menopause-Specific Quality of Life Questionnaire: psychometric evaluation among breast cancer survivors. Menopause 18(3): 289-295. 
106. Mohamed HAE, SM Lamadah, LGA Zamil (2014) Quality of life among menopausal women.International Journal of Reproduction, Contraception, Obstetrics and Gynecology 3(3): 552.

107. Hilditch JR, Lewis J, Peter A, van Maris B, Ross A, et al. (2008) A menopause-specific quality of life questionnaire: development and psychometric properties. Maturitas 24(3): 161-175.

108. Pockaj BA, Gallagher JG, Loprinzi CL, Stella PJ, Barton DL, et al. (2006) Phase III double-blind, randomized, placebo-controlled crossover trial of black cohosh in the management of hot flashes: NCCTG Trial N01CC1. J Clin Oncol 24(18): 2836-2841.

109. Liljegren A, Gunnarsson P, Landgren BM, Robéus N, Johansson H, et al. (2012) Reducing vasomotor symptoms with acupuncture in breast cancer patients treated with adjuvant tamoxifen: a randomized controlled trial. Breast Cancer Res Treat 135(3): 791-798.
110. Newton KM, Carpenter JS, Guthrie KA, Anderson GL, Caan B, et al. (2014) Methods for the design of vasomotor symptom trials: the MsFLASH network. Menopause 21(1) 45-58.

111. Borud EK, Alraek T, White A, Fonnebo V, Grimsgaard S (2007) The effect of TCM acupuncture on hot flushes among menopausal women (ACUFLASH) study: A study protocol of an ongoing multi-centre randomised controlled clinical trial. BMC complement altern medicine $7(1): 6$.

112. Patridge EF, Bardyn TP (2018) Research electronic data capture (REDCap). J Med Libr Assoc 106(1): 142-144.

113. Machin D (2010) Randomized Clinical Trials : Design, Practice and Reporting. $1^{\text {st }}$ ed. PM Fayers and MyiLibrary: Hoboken : Wiley.

114. Gordon HG, Chan AW, Wu P, Vail A, Guyatt GH, et al. (2009) Design, analysis, and presentation of crossover trials. Trials 10(1): 27. 